\title{
APPLICAZIONE DELLA GEOMETRIA SOPRA UNA CURVA ALLA DIMOSTRAZIONE DI UN TEOREMA DI GEISER.
}

\author{
Nota di Eugenio Bertini (Pisa).
}

Adunanza del 27 febbrajo I9ro.

Il teorema di GeIser, che qui si deduce in modo assai semplice da alcuni teoremi generali di geometria sopra una curva, è il seguente:

Ogni curva piana di $4^{\circ}$ ordine e di genere 3 si può ottenere come contorno apparente, sul piano della curva, di una superficie di $3^{\circ}$ ordine, ossia come traccia sul piano stesso del. cono circoscritto a questa superficie da un suo punto ${ }^{x}$ ).

Nei $n^{i}$ I, 2 si dimostra una proprietd che discende facilmente dal cosidetto teorema di riduzione ${ }^{2}$ ) e che forse non era stata esplicitamente osservata, e nei $\mathrm{n}^{\mathrm{i}} 3,4$ se ne trae il detto teorema di GeIser.

I. Sia $C_{n}$ una curva normale di $S_{r}$ di ordine $n$ e genere $p$. Se la serie $g_{n}^{r}$ segnata sopra $C_{n}$ dagli iperpiani di $S_{r}$ è non speciale, o, come dicesi, $C_{n}$ è non speciale, per il ricordato teorema di riduzione, aggiungendo alla $g_{n}^{*}$ un punto qualunque $P_{s}$ si ha una serie incompleta, e quindi $C_{n}$ è proiezione di una $C_{n+1}$ di $S_{r+1}$ da un suo punto semplice $P$, la tangente in $P$ avendo per traccia $P_{1}{ }^{3}$ ). Ciò è ancor vero se $C_{n}$ è speciale, purchè i gruppi canonici contenenti un gruppo di $g_{n}^{r}$ passino tutti per qualche altro punto $P_{1}$ di $C_{n}$. Ma, se ciò non avviene, qualunque punto $P_{1}$ si aggiunga alla detta $g_{n}^{r}$, la serie $g_{n+1}^{r}=g_{n}^{r}+P_{\mathrm{r}}$ è completa. Se non che, a questa $g_{n+1}^{r}$ si puó applicare di nuovo la considerazione precedente, cioè, se è speciale, considerare i gruppi canonici che passano per un suo gruppo, la cui infinita sard inferiore di I a quella dei gruppi canonici passanti per un gruppo di $g_{n}^{r}$, e verificare se quei gruppi hanno qualche punto comune. Se non l'hanno, aggiungendo un qualunque punto $P_{2}$, si ha ancora in

I) GEISER, Veber die Doppeltangenten einer ebenen Curve vierten Grades [Mathematische Annalen, t. I (1869), pp. I29-1 38 ].

2) Cfr.: a) SEGRE, Introduzione alla geometria sopra un ente algebrico semplicemente infinito [Annali di Matematica pura ed applicata, serie II, t. XXII (I894), pp. 4I-142], $\mathrm{n}^{\circ} 86 ; b$ ) BERTINI, La geometria delle serie lineari sopra una curva piana secondo il metodo algebrico [Ibid., serie II, t. XXII (I894), pp. 1-40], $\mathrm{n}^{\mathrm{i}} 20$ e 27; c) Severr, Lezioni di Geometria Algebrica (Padova, Angelo Draghi, 1908), $\mathbf{n}^{\circ} 45$.

3) Cfr. SEGRE, loc. cit. ${ }^{2}$ ) $a, \mathrm{n}^{\circ} 86$. 
$g_{n+2}^{r}=g_{n}^{r}+P_{1}+P_{2}$ una serie completa. E così si puó continuare; ma si arriverà in fine ad una serie

$$
g_{n+k-1}^{r}=g_{n}^{r}+P_{1}+P_{2}+\cdots+P_{k-r}
$$

completa, tale che, o per un suo gruppo non passa alcun gruppo canonico o quelli che vi passano hanno tutti (almeno) un punto $P_{k}$ comune. In amendue i casi (nel primo $P_{k}$ essendo preso arbitrariamente), la serie

$$
g_{n+k}^{r}=g_{n}^{r}+P_{1}+P_{2}+\cdots+P_{k}
$$

é incompleta e peró contenuta in una $g_{n+k}^{r+1}$ completa (giacchè la sua residua rispetto a $P_{k}$ è la $\left.g_{n+k-1}^{r}\right)$. E chiaro che, rispetto alla serie $g_{n+k}^{r+1}$ il gruppo dei punti $P_{1}, P_{2}, \ldots, P_{k}$ è un gruppo neutro, di grado di neutralita I (cioè presentante una sola condizione ai gruppi della serie) ed inoltre che la serie stessa non ha punti fissi, nè è composta. La sua imagine è adunque una $C_{n+k}$ di $S_{r+1}$ con punto $k^{\text {uplo }}$ e si può costruirla così che abbia proprio per proiezione (biunivoca) la $C_{n}$. Si ha quindi la proprietà : Ogni $C_{n}$ normale di $S_{r}$ è proiezione di una $C_{n+k}$ normale di $S_{i+1}$ da un suo punto $k^{\text {uplo }}$ $(k \supseteq \mathrm{I})$. Le $k$ tangenti nel punto $k^{\text {uplo }}$ hanno per traccia i $k$ punti $P_{1}, P_{2}, \ldots, P_{k}$.

2. Suppongasi che $g_{n}^{r}$ sia speciale e indichisi con $i$ il suo indice di specialità : questo si potrd anche dire indice di specialità della stessa $C_{n}$. Se gli $\infty^{i-1}$ gruppi canonici passanti per un gruppo $G$ di $g_{n}^{\tau}$ hanno altri punti comuni e aggiungiamo uno di questi a $G$, si ha, come dicemmo nel $\mathrm{n}^{\circ} \mathrm{I}$, una serie incompleta, ma se aggiungiamo invece un punto $P_{\mathrm{s}}$ diverso da quelli si ha una serie completa speciale. Cosi si ha pure una serie completa speciale aggiungendo un altro punto $P_{2}$ diverso dagli ulteriori punti che abbiano eventualmente a comune i gruppi canonici passanti per $G$ e $P_{1}$ : e cosi si puó continuare, fino a che si giunge ad una

$$
g_{n+i-1}^{r}=g_{n}^{r}+P_{1}+\cdots+P_{i-1}
$$

completa speciale e tale che per un suo gruppo passa un solo gruppo canonico. Aggiungendo un nuovo punto $P_{i}$, diverso dagli eventuali punti di questo gruppo esterni a quello, si ha una

$$
g_{n+i}^{r}=g_{n}^{r}+P_{1}+\cdots+P_{i-1}+P_{i}
$$

completa non speciale e quindi, aggiungendo un nuovo punto $P_{i+1}$ qualsiasi, si ha una serie incompleta $g_{n}^{r}+P_{1}+\cdots+P_{i+1}$ contenuta in una serie completa non speciale $g_{n+i+1}^{r+1}$. Se ne conclude in modo analogo al $\mathrm{n}^{0}$ I che:

Una curva normale $C_{n} d i S_{r}$, di indice di specialità $i$, è proiezione di una curva normale non speciale $C_{n+i+1}$ di $S_{r+1}$ da un suo punto $(i+\mathrm{I})^{\mathrm{uplo}}$.

Si è enunciato il teorema senza distinguere se $C_{n}$ sia speciale o no, perchè, se non è speciale, cioè $i=0$, la proprietd̀ è quella ricordata al principio del $\mathbf{n}^{\circ} \mathbf{I}$. Anzi, applicando tale proprietà successivamente, è manifesto che una curva normale non speciale $C_{n}$ di $S_{r}$ è sempre proiezione di una curva normale non speciale $C_{n+0}$ di $S_{r+\theta}$ da un $S_{\theta-\mathrm{r}}$, qualsiasi $\theta(\mathrm{I})$.

E pure ovvio che per una $C_{n^{\prime}}$ (non normale) di $S_{r^{\prime}}$, la quale sia proiezione di una $C_{n}$ normale di $S_{r}$ da un $S_{r-r_{-1}^{\prime}}$ valgono le proposizioni precedenti e quella del $\mathrm{n}^{0} \mathrm{I}$, 
soltanto assumendo per spazî di proiezione rispettivamente spazî $S_{r-r^{\prime}}, S_{r-r^{\prime}+\theta_{-1}}$, passanti rispettivamente per gli spazî di proiezione ivi indicati.

Infine si noti che nella proposizione del $\mathrm{n}^{\circ}$ I si ha $k \leq i+\mathrm{I}$ e che il più piccolo valore di $k$, quando $C_{n}$ è speciale, si puó fissare nei singoli casi in base alle considerazioni fatte in quel $\mathrm{n}^{\circ}$. Cosi una curva piana di $6^{\circ}$ ordine $C_{6}$ con un punto triplo e due punti doppî $(p=5)$ è proiezione di una $C_{7}$ speciale di $S_{3}$ da un suo punto semplice, per il teorema del $\mathrm{n}^{\circ} \mathrm{I}^{4}$ ), ed è proiezione di una $C_{8}$ non speciale di $S_{3}$ da un suo punto doppio per il teorema di questo $\mathrm{n}^{\circ}$.

3. Considerisi ora una $C_{4}$ piana di genere 3. La sua serie canonica, che è la $g_{4}^{2}$ segnata dalle rette del piano, accresciuta di un punto qualunque $P_{\mathrm{I}}$, dà una serie

$$
g_{5}^{2}=g_{4}^{2}+P_{\mathrm{I}}
$$

completa non speciale: cosicchè, aggiungendo un nuovo punto qualunque $P_{2}$, la

$$
g_{4}^{2}+P_{t}+P_{2}
$$

è incompleta e contenuta in una serie completa $g_{6}^{3}$. Si ha adunque $\left(\mathrm{n}^{0} 2\right)$ che la $C_{4}$ è proiezione di una $C_{6}$ di $S_{3}$, avente punto doppio $P$, da questo punto. La $C_{6}$ è l'imagine della $g_{6}^{3}$ segnata su $C_{4}$ dalle coniche per $\mathrm{i}$ punti $P_{3}, P_{4}$ in cui la retta $P_{1} P_{2}$ sega ulteriormente la $C_{4}$ stessa (per il teorema del Resto).

La detta $C_{6}$ giace sopra una quadrica $\mathbf{s}_{2}$, perchè le quadriche di $S_{3}$ passanti per il suo punto doppio $P$ segnano su $C_{6}$ una $g_{\text {so }}$, non speciale, che puó essere quindi al più di dimensione 7. Anzi, potendo passare per $C_{6}$ una sola quadrica, si vede che la detta serie è precisamente una $g_{\mathrm{I} 0}^{7}$. Siccome poi nella proiezione stereografica di $\Sigma_{2}$ da $P$ i punti $P_{1}, P_{2}$ corrispondono alle direzioni di $C_{6}$ uscenti da $P$, saranno $P_{3}, P_{4}$ i punti fondamentali della detta proiezione stereografica, cioè $P P_{3}, P P_{4}$ generatrici di $\Sigma_{2}$.

Per la stessa $C_{6}$ passano $\infty^{4}$ superficie di $3^{\circ}$ ordine $\Sigma_{3}$ (almeno), perchè quelle che passano per il punto doppio segnano una $g_{\mathrm{I} 6}$ non speciale e quindi di dimensione $\mathrm{I} 3$ (al piì).

4. Si prendano $P_{1}, P_{2}$ nei punti di contatto di una tangente doppia di $C_{4}$ : onde $P_{3}, P_{4}$ cadono negli stessi punti. Allora le tangenti alla $C_{6}$, che ne risulta, nel suo punto doppio $P$, sono le generatrici di $\Sigma_{2}$ passanti per $P$, e quindi sono anche le rette osculatrici di ogni $\mathbf{\Sigma}_{3}$ (passante per $C_{i}$ ), perchè un piano variabile intorno ad una di esse sega $\Sigma_{3}$ in una cubica passante per i 6 punti in cui il piano taglia $C_{6}$ : ma di questi, tre cadono in $P$ sulla retta stessa (e gli altri tre sull'altra retta che forma con essa l'intersezione del piano con $\Sigma_{2}$ ); e petó, etc.

Ora si afferma che fra le infinite $\Sigma_{3}$ suddette si puó scegliere una rispetto a cui $\Sigma_{2}$ sia quadrica polare di $P$, cosicchè allora $C_{6}$ sarà curva di contatto del cono circo. scritto a quella $\Sigma$, da $P$, e il teorema di GEISER sara dimostrato. In vero, si prendano su $C_{6}$ quattro punti generici (e basta che non sieno in un piano) e si determini una

4) Riapplicando questo stesso teorema alla $C_{7}$ si trova che $C_{6}$ è proiezione di una $C_{8}$ (canonica) di $S_{4}$ da una sua corda.

Rend. Circ. Matem. Palermo, t. XXX (20 sem. I9ro). - Stampato ii 24 marzo $x 910$. 
$\Sigma_{3}$ imponendo le ulteriori condizioni che essa abbia in ciascuno di quei punti per piano tangente il piano che proietta da $P$ la tangente a $C_{6}$ nel punto stesso. La quadrica polare di $P$ rispetto a quella $\Sigma_{3}$ ha per generatrici partenti da $P$ le rette osculatrici della $\Sigma_{3}$ e per conseguenza ha comuni con $\Sigma_{2}$ queste rette e inoltre i detti quattro punti, e però coincide con $\mathbf{\Sigma}_{2}$.

Si puó notare che, non potendo manifestamente essere $\Sigma_{2}$ quadrica polare di più $\Sigma_{3}$ passanti per $C_{6}$, l'infinita di queste è proprio 4 , ossia la serie segnata su $C_{6}$ dalle superficie di $3^{\circ}$ ordine per $P$ è una $g_{16}^{\mathrm{r}^{3}}{ }^{5}$ ).

Pisa, I2 febbrajo IgIo.

E. BERTINI.

5) Cio, del resto, come pure l'essere $g_{10}^{7}$ la serie segnata su $C_{6}$ dalle quadriche per $P$, è caso particolare di un teorema noto \{cfr. BerTINI e SEveri, Osservazioni sul Restsatz per una curva iperspa. ziale [Atti della R. Accademia delle Scienze di Torino, Classe di Scienze Fisiche, Matematiche e Naturali, vol. XLIII (1907-I908), pp. 439-447]: Lettera del Prof. E. Bertini al Prof. F. SeverI (pp. 439-444), I. (p. 440)\}. 